\title{
Cognitive-Femtocell Based Resource Allocation in Macrocell Network
}

\author{
Joydev Ghosh*, Dushantha Nalin K. Jayakody ${ }^{\dagger}$, and Marwa Qaraqe ${ }^{\dagger}$ \\ * School of Computer Science and Robotics, National Research Tomsk Polytechnic University, RUSSIA \\ ${ }^{\dagger}$ ICT Division, College of Science and Engineering, Hamad Bin Khalifa University, \\ Qatar Foundation, QATAR \\ Email: joydev.ghosh.ece@gmail.com,nalin.jayakody@ieee.org and mqaraqe@hbku.edu.qa
}

\begin{abstract}
In this paper, we articulate the network coverage issues for both Femto Users (FUs) and Macro Users (MUs) located at cell edges. The cognitive-femtocell networks functioning under the vicinity of a macrocell frontier where the parameters such as pathloss, shadowing, Rayleigh fading have considered into the system model. The users, located at network border are positioned far apart from the Macro Base Station (MBS). This can be treated as the underprivileged users. The underprivileged users are to be facilitated by the femto cell base stations to provide uninterrupted QoS. We present on the overall outage probability of Single Input single Output (SISO) users and Single Input Multiple Output (SIMO) users, respectively, by taking several circumstantial components such as such as probability density function (PDF), location gap between base stations (BSs) and users, intra-tier interference and inter-tier interference into account. Further, evaluation has been extended by considering network throughput as the efficiency measures based on the sub-carrier and the power allotment in the dual tier network.
\end{abstract}

\section{INTRODUCTION}

The radio frequency spectrum has always been an important issue of wireless communication because of increasing demands of high data rate frequency bandwidth. It has been observed that a macrocell could not provide the quality of service to the indoor user due to inability to penetrate through walls. Femtocells are low power base stations that have converted the centralized cellular network into distributed networks [1]. The access point of the femtocell is known as the femto access point (FAP). It improves coverage, quality of service, and reduces battery draining due to short range transmissions. The spectrum can be managed in two ways: licensed spectrum i.e. a particular portion of band has been allotted for organizations, the devices with licenses allow to perform communication within the allotted band with negligible interference [2]. Unlicensed spectrum also known as free or open spectrum which require neither license, nor central control for users.

The key properties and capability of the Orthogonal Frequency Division Multiple Access (OFDMA) technique fabricates it absolutely perfect for the cognitive radio based communication networks. The $\mathrm{CR}$ network

978-1-5386-3531-5/17/\$31.00 (c) 2017 IEEE architecture has two types of networks: primary network where the users possess the privilege to access the spectrum as they hold licenses and secondary network where the users use the band only when the primary users (PUs) are not present. In OFDMA network, secondary users (SUs) can share the spectrum provided that primary network should not impose appreciable interference. The discussed idea of spectrum sharing is analogous to the implementation of femtocell in macrocell environment.

The use of Fractional frequency reuse (FFR) in future generation heterogenous networks builds trade-off between data speed and network service of users located at macrocell boarder area [9], average cell capacity and energy efficiency. In this work, we mainly focus on FAP due to the following reasons:(i) The significance of the inter-cell interference has been increased for FAPs due to the large difference of transmit power gap between the FBSs and MBS and (ii) Introduction of FAPs can nulify the effect of an underprivileged users on the entire network performance.

The main contributions of the paper are as follows:

1) An extricated system model for resource allocation in the dual layer heterogeneous network has been put in place.

2) Numerical results has been presented to assess the performance of underprivileged users ${ }^{1}$

\section{SySTEM MODEL}

We use a network model with femtocells installed under the network coverage of MBS and it co-exists with macrocell. In this system model, one macrocell and four femtocells are deployed to quantify the DL communications. As depicted in Fig. 1, a MBS is located at the center of the network having 4 FBSs distributed within the macrocell coverage at $(2,2),(-2,2),(-2,-2)$, $(2,-2)$. In a macrocell, $N_{M U E}$ number of user equipment (UE) in totality is uniformly spread within the area of the network coverage.

Let us consider MBS be the BS located at macrocell centre and and $M U^{(u)}$ be the $u^{t h}$ portable $\mathrm{MU}$ in its

\footnotetext{
${ }^{1}$ The user located at macrocell edge area is an outage user, the more precisely underprivileged user, who is not getting standard QoS usually.
} 


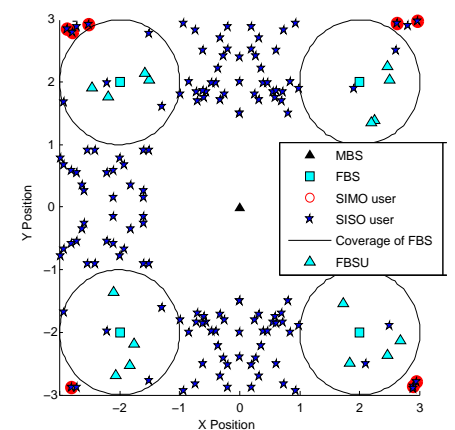

Fig. 1. A co-existence scenario of FBS and MBS of the proposed network

network coverage. Similarly, total $N_{F U}$ is a total number of FUs that are randomly distributed in the $j^{\text {th }}$ femtocell, $j \in 1,2, \cdots, N_{F}$ and let $F A P_{j}$ and $F U E_{j}^{(i)}$ be the $j^{t h}$ FAP and the $i^{t h}$ user in the $j^{\text {th }}$ femtocell, respectively. The resource blocks (RBs) are uniformly among the MUs and the identical resources are reutilized within each FBS.

FBSs opportunistically re-utilzed RBS allocated to other cells for optimum dynamic cell loading if the required average user throughput is not achieved with the firstly alloted RBs. We further consider that in the entire network there are a total $N_{R B}$ number of RBs. In this system model, the matter of greatest importance is to decide the number of RBs (i.e., $N_{M R B}$ ) by the macrocell to serve its own UEs in the network. The remaining unassigned RBs, i.e., $\left(N_{R B}-N_{M R B}\right)$, are orthogonally alloted to FBSs, thus, the number of RBs that are alloted to each Femto cell is $\left[\frac{N_{R B}-N_{M R B}}{N_{F}}\right]$.

\section{A. Path Loss Model:}

Macrocell Path Loss Model: Here we adapt Okumura-Hata propagation model. This accounts urban area as a reference and by the consideration of correction factors, we get:

$$
L_{d B}=A+B \log _{10}(d)-E,
$$

where, $A=69.55+26.16 \log _{10} f_{c}-13.82 \log _{10} h_{b}$, $B=44.9-6.55 \log _{10} h_{b}, E=$ $3.2\left(\log _{10}\left(11.7554 h_{m}\right)\right)^{2}-4.97$ for large cities, $f_{c} \geq 300 M H z$

Femtocell Path Loss Model Different path loss exponents and breakpoint distance of $100 \mathrm{~m}$ are considered to characterize path loss model of Femto cell [2] as below:

$$
L=10 \alpha_{p 1} \log _{10} r+L_{1}, \quad \text { for } \quad r<r_{b},
$$$$
=10 \alpha_{p 2} \log _{10} \frac{r}{r_{b}}+10 \alpha_{p 1} \log _{10} r_{b}+L_{1} \quad \text { for } \quad r>r_{b} \text { by: }
$$

where, $L_{1}=$ Reference Path Loss at $r=1 \mathrm{~m}$, $r_{b}=$ Breakpoint distance, $\alpha_{p 1}=$ Pathloss exponent for $r \leq$ $r_{b}, \alpha_{p 2}=$ Pathloss exponent for $r>r_{b}$.
TABLE I

VARIABLES RELATED TO OKUMURA-HATA MODEL [10]

\begin{tabular}{|l|l|l|}
\hline Parameters & Symbol & Range \\
\hline Carrier Frequency & $f_{c}$ & $150 \leq f_{c} \leq 2000(\mathrm{MHz})$ \\
\hline BS Antenna Height & $h_{b}$ & $30 \leq h_{b} \leq 200(\mathrm{~m})$ \\
\hline MS Antenna Height & $h_{m}$ & $1 \leq h_{m} \leq 10(\mathrm{~m})$ \\
\hline $\begin{array}{l}\text { Distance Between BS } \\
\text { and MS }\end{array}$ & $d$ & $1 \leq d \leq 20(\mathrm{~m})$ \\
\hline
\end{tabular}

To neglect high transition between the two regions:

$$
L=L_{1}+10 \alpha_{p 1} \log _{10} r+10\left(\alpha_{p 2}-\alpha_{p 1}\right) \log _{10}\left(1+\frac{r}{r_{b}}\right) .
$$

\section{B. Channel Model}

The locations of MBS, MU, FBS, FU are depicted in Fig. 1. Here, MU accounts as PU and FU accounts as SU. The connection gain between MBS and $u^{t h}$ user can be given by:

$$
G_{p 2 p, u}\left(r_{u}, \theta_{u}\right)=d_{u}^{\left(-\alpha_{p}\right)} 10^{\frac{\zeta_{s}}{10}}\left|h_{p 2 p, u}\right|^{2},
$$

where $d_{u}\left(r_{u}, \theta_{u}\right)$ represents the separation gap of MU at a location $\left(r_{u}, \theta_{u}\right)$ to MBS and $\left|h_{p 2 p, u}\right|^{2}$ indicates the channel gain of MBS to its associated MU. In addition, the deployment of FBSs are made in such a way that it attenuates all issues of network coverage overlapping of FBSs. The connection gain between $j^{\text {th }}$ FBS and $i^{t h}$ FU can be given by:

$$
G_{s 2 s, j i}=d_{s 2 s, j i}^{-\alpha_{p}} 10^{\frac{\zeta_{s}}{10}}\left|h_{s 2 s, j i}\right|^{2}
$$

where $d_{s 2 s, j i}$ represents separation gap between $j^{\text {th }}$ FBS and $i^{\text {th }} \mathrm{FU}, \zeta_{s}$ (in $\mathrm{dB}$ ) denotes Gaussian random variable with zero mean and variance, $\sigma^{2}$ due to shadowing in the channel, and $\left|h_{s 2 s, j i}\right|^{2}$ indicates the channel gain of $j^{t h}$ FBS to its associated $i^{t h}$ FU. A FU is considered to be located within the round shape network coverage of radius, $r_{f}$. If the location of a FBS is $\left(r_{j}, \theta_{j}\right)$ and the location of a FU is $\left(r_{i}, \theta_{i}\right)$. Then,

$$
d_{s 2 s, j i}=\sqrt{\left(r_{j} \cos \theta_{j}-r_{i} \cos \theta_{i}\right)^{2}+\left(r_{j} \sin \theta_{j}-r_{i} \sin \theta_{i}\right)^{2}} .
$$

If the location of a MBS is $\left(r_{k}, \theta_{k}\right)$, then the distance of it from FU can be given by:

$$
d_{p 2 s, k i}=\sqrt{\left(r_{k} \cos \theta_{k}-r_{i} \cos \theta_{i}\right)^{2}+\left(r_{k} \sin \theta_{k}-r_{i} \sin \theta_{i}\right)^{2}} .
$$

The link gain from $k^{t h}$ MBS to $i^{\text {th }}$ FU can be given by:

$$
G_{p 2 s, k i}=d_{p 2 s, k i}^{-\alpha_{p}} 10^{\frac{\zeta_{s}}{10}}\left|h_{p 2 s}\right|^{2},
$$

where $d_{p 2 s, k_{i}}$ represents the location gap between $k_{t h}$ MBS to $i_{t} h$ FU. An assumption has been made that the channels have a coherent time greater than or equal to 


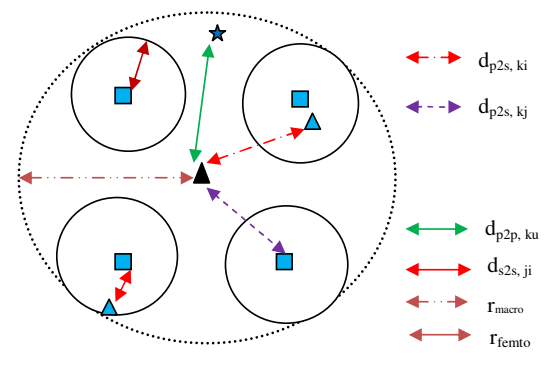

Fig. 2. Computation of distance/path from the position of a user (PU/MBSU or SU/FBSU) to MBS and FBS.

a time slot and all the channels gains are independent. The channel gain between $k^{t h} \mathrm{MBS}$ and its associated $i^{\text {th }}$ FBSU denotes by the $\left|h_{p 2 s, k i}\right|^{2}$. Rayleigh fading is included with shadowing and pathloss for analysis and Rayleigh fading gives controllable results that help to understand system response to a particular situation. Moreover, the notation $x$ has been used to denote the serving network entity for a generic user. Therefore, if a user is linked to a FAP, then $x=a$, and if a user is linked to a MBS, then $x=b$. The analysis is conducted on a typical user located at the origin without any loss of generic laws [5]. Thus, the signal to interference plus noise ratio (SINR), $\gamma_{x}$ at the typical user locates in the origin (also which holds for any generic user) served by FAP or MBS is given by

$\frac{\left.P_{x} D_{x}^{-\alpha_{p}}\left|h_{x}\right|^{2}\right)}{\sum_{b_{i} \in \bar{\psi}_{b_{x}}} P_{b} b_{i}^{-\alpha_{p}}\left|h_{b_{i}}\right|^{2}+\sum_{a_{i} \in \bar{\psi}_{a_{x}}} P_{a} a_{i}^{-\alpha_{p}}\left|h_{a_{i}}\right|^{2}+N}$,

where $D_{x}$ is the distance from the user to the serving network entity (i.e. an MBS or a FAP), $\psi \approx b_{x}$ denotes the set of interfering MBSs, $\psi \approx a_{x}$ denotes the set of interfering FAPs and $N$ is the noise power [6]. Here $P_{x}$ is designated as the proportion of total transmit power on a given serving network entity. Likewise, the channel gains from a generic location $x \in D^{2}$ to the MBS, $b_{i}$ and the FAP, $a_{i}$ are denoted by $h_{b_{i}} \approx \sqrt{X_{b_{i}}^{2}+Y_{b_{i}}^{2}}$ and $h_{a_{i}} \approx$ $\sqrt{X_{a_{i}}^{2}+Y_{a_{i}}^{2}}$, respectively, where $X_{x}, Y_{x}$ are independent Gaussian random variables with zero mean and desired variance.

\section{Outage Probability for DL Transmission of Macro User $(M U)$ :}

An FAP encountered two kinds of outage events; one is channel unavailability due to opportunistic channel access and other one is due to SINR outage. The outage probability of a SISO or SIMO may be expressed as:

$$
P_{\text {out }, S I S O}=\operatorname{Prob}_{x} \leq S I N R_{t h d(S I S O)} .
$$

$$
P_{\text {out }, S I M O}=\operatorname{Prob}_{x} \leq S I N R_{\text {thd(SIMO })} .
$$

\section{Throughput of Macrocell Network}

We assume that exact synchronization in time and frequency domain, thus, interference among the surrounding or nearest RBs is neglected. The reachable throughput, $T_{p}$ of an UE can be calculated from Shannons theorem. We have

$$
T_{p}=W \log _{2}\left(1+\gamma_{x}\right),
$$

where ${ }_{x}$ Signal to Interference plus Noise Ratio, and $W$ is allocated bandwidth to macrocell networks.

\section{Simulation MODEL}

The simulation variables specified in Table 1 and Table 2 have been used here. For simplicity of analysis, we accounts the pathloss only. The generation of the users' positions and interference powers are carried out considering the following steps.

1. A constant number of users $\left(N_{U E}=N_{M U E, u}+\right.$ $\left.N_{F U E, j i}\right)$ is generated. They are randomly distributed within the coverage area. This includes all MBSUs or PUs $\left(N_{M U E, u}\right)$ and FBSUs or SUs $\left(N_{F U E, j i}\right)$. Here, $j \in 1,2, \cdots, N_{F} ; m=60, u 1,2,3, \cdots, m R_{b}, R_{b} \in$ No. of resource block (RB); $i 1,2,3, . n, n \in$ any large integer value.

2. The locations (in the ( coordinate system) of all SUs and PUs $N_{U E}$ are generated within the coverage of macrocell. $N_{F U E, j i}$ number of SU receivers are generated around $j$ number of corresponding SU transmitters (FBS/FAP). The SU receivers are present around a SU transmitter, within a circle of radius, $r_{\text {femto }}$.

3. For each of the NUE users, the link gains corresponding to $\mathrm{BS}$ and $\mathrm{UE}$ are generated, following the formulas derived in Section II.

4. The received signal strength (RSS) is evaluated from PU/MBSU or SU/FBSU at the reference MBS or FBS. Similarly, interference power at a PU receiver or $\mathrm{SU}$ receiver is evaluated, considering all the possibilities.

5. Next, the SINR for a PU or Macro user and a SU or Femto user are computed.

\section{A. Probability of Outage for MBSUs and FBSUs}

The following steps are considered.

1. The SINR is generated for a desired user as shown in the previous section and compared with a threshold value given by $S I N R_{t h d(S I S O)}$ and $S I N R_{\text {thd(SIMO) }}$.

2. If the $\gamma_{x}$ falls below $S I N R_{\text {thd(SISO) }}$, an outage counter (outage count(SISO) $)$ is incremented. Similarly, If the ${ }_{x}$ falls below $S I N R_{t h d(S I M O)}$, an outage counter (outage count (SIMO) $)$ is incremented.

3. Steps (2) and (3) are repeated a large $\left(N_{t}>>1\right)$ number of times to yield an accurate estimate of the 


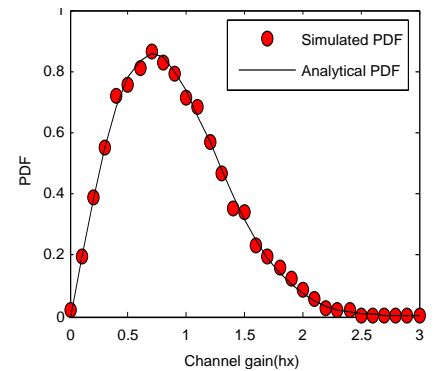

Fig. 3. Contrast of PDF in simulated and analytical response

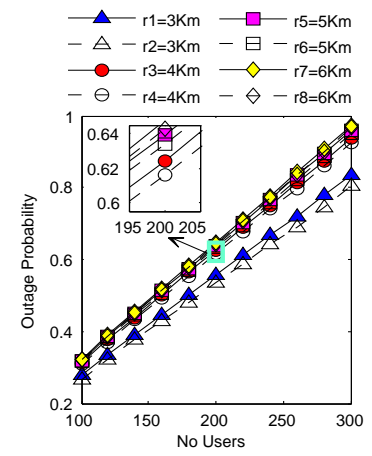

Fig. 4. Probability of outage for SISO users vs. the number of UEs

probability of outage as $P_{\text {out }, \text { SISO }}=\left(\frac{\text { outage }_{\text {count }(\text { SISO }}}{N_{t}}\right)$ and $P_{\text {out }, S I M O}=\left(\frac{\text { outage }_{\text {count }(S I M O)}}{N_{t}}\right)$. Please note that outage probabilities for a MBSU and a FBSU are evaluated in the same way.

\section{Results AND Discussion}

The main parameters of the simulation framework are set as shown in Table 1 and Table 2, and separately simulated outcome of Rayleigh fading element is probed by comparing it with theoretical response obtained from Rayleigh fading equation [8] prior to the inclusion of it into the networks.

In Fig.3, the simulated pdf from $X_{x}, X_{y}$ samples and theoretical PDF obtain from the Rayleigh fading

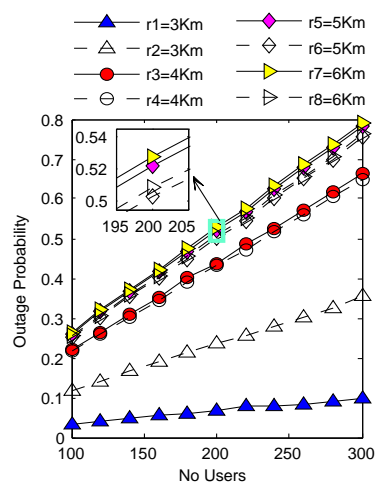

Fig. 5. Probability of outage for SIMO users vs. number of UEs

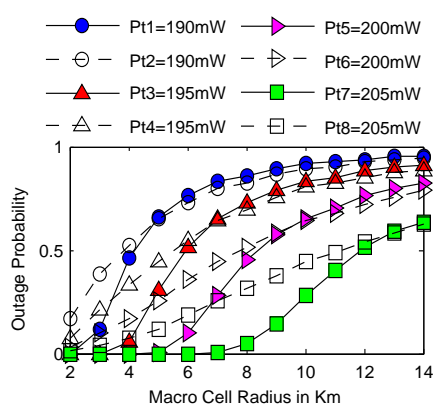

Fig. 6. Probability of outage for SIMO users vs. number of UEs

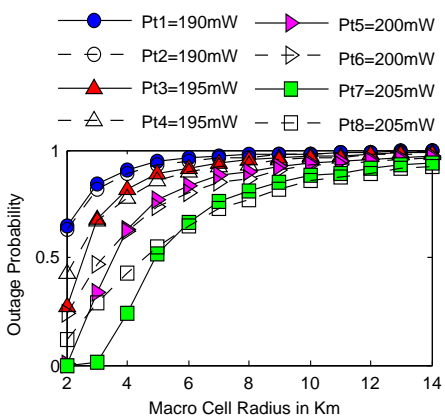

Fig. 7. Outage probability for SIMO users vs number of users

equation are compared and from there inclusion of the Rayleigh fading element in the environment is validated. $r_{1}, r_{3}, r_{5}, r_{7}$ : The environment included pathloss and shadowing only. $r_{2}, r_{4}, r_{6}, r_{8}$ : The environment included pathloss, shadowing and Rayleigh fading.

In Fig. 4 and 5, the outage probability for SISO users and SIMO users are are depicted as a function of the number of users, either MU or FU. It can be noticed that the probability of outage for either SISO or SIMO users

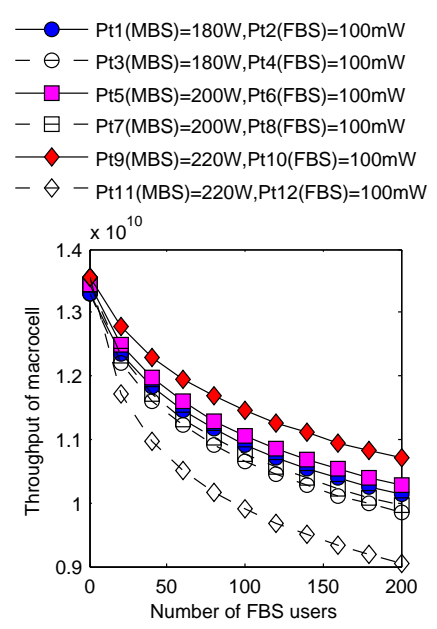

Fig. 8. Probability of outage for SIMO users vs. number of UEs 


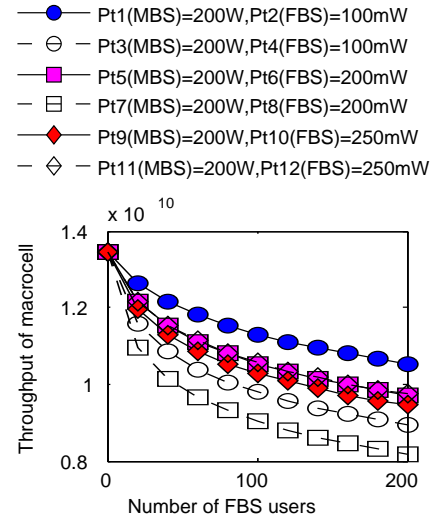

Fig. 9. Probability of outage for SIMO users vs. number of UEs

grows for the growing number of users, either MUs or FUs, and there are similarities and dissimilarities in results between the environment which added pathloss and shadowing only, and the environment in which additionally Rayleigh fading included. $P_{t 1}, P_{t 3}, P_{t 5}, P_{t 7}, P_{t 7}$ : The environment included pathloss and shadowing only. $P_{t 2}, P_{t 4}, P_{t 6}, P_{t 8}:$ The environment included pathloss, shadowing and Rayleigh fading included. This is happening because of the interference generated due to rapidity of the multiple access. However, the outage probability decreases for the consideration of less number of MU. Also, it can be noticed that outage probability of SISO and SIMO users grow with the enhancement of coverage radius of MBS keeping antenna transmitting power of BS fixed. In Fig. 5, as we can observe, the minimum outage probability of SIMO user is recorded at MBS coverage radius of $3 \mathrm{Km}$. However, there is no noticeable change with respect to the growing number of users.

In Fig. 6 and Fig. 7, all the FBSs are considered to have uniform transmitting power. We have also illustrated the influence of different FBS transmitting power, keeping MBS transmitting power fixed, on outage probability. The result shows that, quite a few instances when MBSU suffering from FBS interference due to implantation of FBS, but at the same time FBS provides network coverage which helps to reduce overall outage of the networks. Normally, any macrocell user (MBSUs) who experiences/faces interference would be switched to a different channel and would avoid outage from the femtocells. The probability of outage for a SISO or SIMO user is higher when macrocell radius is higher. However, the probability of outage of a SISO or a SIMO user does not change much with distance beyond $16 \mathrm{~km}$.

In Fig. 8 and Fig. 9, throughput of macrocell network for SISO and SIMO users are shown as a function of the number FBSUs. Throughput of macrocell network decreases with increase in number of FBSUs. Three different values of antenna transmit power for UEs are considered in this analysis. The minimal reaction of this net- work is explored in Fig. 8 and 9 for two different uptake environments, one without Rayleigh fading used in [7] whereas another one with Rayleigh fading. In Fig. 8, the measure of throughput performance is taken by assuming different MBS transmit power, while keeping FBS power fixed. Fig. 9 highlights the throughput performance by considering different FBS transmit power, while keeping MBS power fixed $P_{t 1}, P_{t 2}, P_{t 5}, P_{t 6}, P_{t 9}, P_{t 10}$ : The environment included pathloss and shadowing only. $P_{t 3}, P_{t 4}, P_{t 7}, P_{t 8}, P_{t 11}, P_{t 12}:$ The environment included pathloss, shadowing and Rayleigh fading.

\section{CONCLUSIONS}

In this work, we introduced a simulation model to study the outage impact in cognitive-femtocell deployed macrocell network. We introduced an extricated network model where the variation of network behavior is compared for two different uptake environments, one by adding pathloss and shadowing only whereas the second one by additionally including the Rayleigh fading with other two parameters. The overall outage performance of SISO and SIMO user equipment (UE) becomes better as their (FBS) transmission power enhances. For the given scheme, our results suggest that the throughput depends strongly on the intensity and transmission powers of FAPs; whereas antenna transmitting power of MBS has a limited impact.

\section{REFERENCES}

[1] S. Cheng, S. Lien, F. hu, and K.Chen,"On exploiting cognitive radio to mitigate interference in macro/femto heterogeneous Networks," IEEE Wireless Commun., vol.18, no.3, pp. 40-47, June 2011.

[2] 3GPP TS36.300 V9.0.0,"Evolved universal terrestrial radio access (EUTRA) and evolved universal terrestrial radio access network (EUTRA)," 2009.

[3] Sylvain Ranvier, "Path loss models: S-72.333 Physical layer methods in wireless communication systems," Helsinki University of Technology, Smarad Centre of Excellence, 23 November 2004.

[4] Ian Wassell and Yan Wu,"Radio Propagation Modelling," University of Cambridge Computer Laboratory.

[5] J. Andrews, F. Baccelli, and R. Ganti,"A tactable approach to coverage and rate in cellular network," IEEE Transaction on Communications, vol.59, no.11, pp.-3122-3134, November 2011.

[6] M. Haenggi and R. Ganti,"Interference in large wireless networks," in Foundations and Trends in Networking. now publishers, 2008, vol.3, no.2, pp.127-248.

[7] J. Ghosh, and S. Dhar Roy,"Mitigating ICI at cell edges in cognitive-femtocell networks through fractional frequency reuse," International Journal of Communication Networks and Distributed Systems, Volume 16, Issue 2, pp.162-175, Feb 2016.

[8] M. M. Siddiqui,"Some Problems Connected with Rayleigh Distributions," JOURNAL OF RESEARCH of the National Bureau of Standards-D. Radio Propagation Vol. 66D, No.2, April 1962.

[9] J Ghosh, S Dhar Roy,"Qualitative analysis for coverage probability and energy efficiency in cognitive-femtocell networks under macrocell infrastructure," Electronics Letters, vol.51, issue 17 @ IET 2015.

[10] D. Lopez-Perez, A. Valcarce, G. De La Roche, and J. Zhang, "OFDMA femtocells: a roadmap on interference avoidance," IEEE Communications Magazine, Vol. 47(9), 2009, pp. 4148. 